\section{SAT0253 EFFICACY AND SAFETY OF LEFLUNOMIDE THERAPY IN LUPUS NEPHRITIS:A META-ANALYSIS}

Q. Ren ${ }^{1}$, H. Zeng ${ }^{2} .{ }^{1}$ Department of Pediatric Allergy, Immunology and Rheumatolog; ' 2 Department of Pediatric Allergy, Immunology and Rheumatology, Guangzhou Women and Children's Medical Center, Guangzhou, China

Objectives: To evaluate the efficacy and safety of leflunomide in treating lupus nephritis (LN).

Methods: According to the requirements of meta-analysis, a literature search about efficacy and safety of leflunomide therapy in LN was performed among Cochrane clinical controlled trials database, PubMed, BMJ-Clinical Evidence, CNKI, VIP and Wanfang data from the establishment of the database till December 2010.All included RCTs were graded in term of randomization, allocation concealment and blinding and non-RCTs were graded in term of grouping method, blinding, withdrawal and loss of follow-up, baseline comparability, diagnostic criteria and bias control. RevMan 5.0 software was used for metaanalysis.

Results: A total of 828 literatures were included. Five RCTs and 2 nonRCTs were enrolled for meta-analysis.Leflunomide group was treated with leflunomide and glucocorticoid, while control group was given cyclophosphamide and glucocorticoid or placebo. The $24 \mathrm{~h}$ urine protein, SCr and SLEDAl scores of leflunomide group were significantly lower than that of cyclophosphamide group. There was no significant difference in $\mathrm{C} 3$,positive rate of anti-ds DNA between leflunomide group and cyclophosphamide group. There was no significant difference in infection, herpes zoster,hypertension, palpitations, leukopenia, alopecial, elevation in ALT, memoxenia, rashes, or the incidence of gastrointestinal reactions between the two groups.

Conclusions: Based on the current evidence, the efficacy and safety of leflunomide for treatment of $L N$ are close to cyclophosphamide. Further evidence from RCT studies is needed to elucidate the efficacy and safety of leflunomide for LN

Disclosure of Interest: None declared

DOI: 10.1136/annrheumdis-2017-eular.2051

\section{SAT0254 SAFETY, TOLERABILITY, AND PHARMACOKINETICS OF SUBCUTANEOUS AND INTRAVENOUS ANIFROLUMAB IN HEALTHY VOLUNTEERS}

R. Tummala ${ }^{1}$, T. Rouse ${ }^{2}$, A. Berglind ${ }^{2}$, L. Santiago ${ }^{3} \cdot{ }^{1}$ AstraZeneca,

Gaithersburg, MD, United States; ${ }^{2}$ AstraZeneca, Gothenburg, Sweden;

${ }^{3}$ Medlmmune, Mountain View, CA, United States

Background: Anifrolumab is a fully human anti-interferon- $\alpha$ receptor monoclonal antibody in Phase III development for systemic lupus erythematosus (SLE). In Phase llb trials, intravenous (IV) anifrolumab (300 mg every 4 weeks) significantly decreased SLE disease activity with safety and tolerability comparable to placebo. ${ }^{1}$ Objectives: The primary objective of this Phase I, double-blind, randomized, controlled study (NCT02601625) was to characterize the pharmacokinetics (PK), safety, and tolerability of anifrolumab administered subcutaneously (SC) and intravenously to healthy volunteers.

Methods: Thirty male and female adults were assigned to three sequential treatment cohorts of equal size (anifrolumab $300 \mathrm{mg} \mathrm{SC}$ injection; anifrolumab $300 \mathrm{mg}$ IV; anifrolumab $600 \mathrm{mg} \mathrm{SC}$ by infusion). Individuals were randomized within each cohort to receive a single dose of either anifrolumab ( $n=6 /$ cohort) or placebo (PBO) ( $n=4 /$ cohort). Serial blood samples were collected up to Day 85. Serum anifrolumab concentrations were analyzed with a validated assay. PK parameters were estimated by noncompartmental analysis. Immunogenicity of anifrolumab was assessed by measuring serum anti-drug antibodies (ADAs).

Results: Anifrolumab serum concentration-time profiles and primary PK parameters in healthy volunteers are presented in the figure and table, respectively. Anifrolumab serum concentrations were below the limit of detection in all individuals by 85 days post dose. Maximum serum concentrations in the SC cohorts occurred after 4 to 7 days. Exposure to SC anifrolumab increased approximately dose proportionally from $300 \mathrm{mg}$ to $600 \mathrm{mg}$ based on AUC. At the $300-\mathrm{mg}$ dose, anifrolumab exposure after SC administration reached approximately $86 \%$ of the IV administration exposure. SC administration of anifrolumab $300 \mathrm{mg}$ and PBO elicited minimal injection-site reactions. Transient injection-site induration

Anifrolumab Serum PK Parameters

\begin{tabular}{lccc}
\hline & \multicolumn{3}{c}{ Anifrolumab } \\
\cline { 2 - 4 } & $300 \mathrm{mg} \mathrm{SC}(\mathrm{N}=6)$ & $300 \mathrm{mg} \mathrm{IV}(\mathrm{N}=6)$ & $600 \mathrm{mg} \mathrm{SC}(\mathrm{N}=6)$ \\
\hline $\mathrm{C}_{\text {max }}(\mu \mathrm{g} / \mathrm{mL})$ & & & \\
$\mathrm{Mean} \pm \mathrm{SD}$ & $36.2 \pm 11.6$ & $82.4 \pm 13.2$ & $63.9 \pm 20.5$ \\
$\mathrm{CV}(\%)$ & 32.1 & 16.0 & 32.0 \\
$\mathrm{AUC}($ day. $\mu \mathrm{g} / \mathrm{mL})$ & $785 \pm 331$ & $907 \pm 175$ & $1,828 \pm 680^{\mathrm{a}}$ \\
$\quad$ Mean $\pm \mathrm{SD}$ & 42.1 & 19.3 & 37.2 \\
$\mathrm{CV}(\%)$ & 4.13 & 0.03 & 7.00 \\
$\mathrm{t}_{\text {max }}$ (day) & $4.02-7.00$ & $0.03 ? 1.03$ & $3.96 ? 8.95$ \\
$\quad$ Median & &
\end{tabular}

${ }^{a} \mathrm{~N}=5$ because 1 individual was lost to follow-up at 1 month post dose. AUC = area under serum concentration-time curve from time zero extrapolated to infinity; $\mathrm{C}_{\max }=$ observed maximum serum concentration; $\mathrm{CV}=$ coefficient of variation; $\mathrm{t}_{\max }=$ time to reach maximum serum concentration. occurred in five of six individuals in the anifrolumab 600-mg group and two of four in the PBO group. Transient, mild to moderate injection-site induration and pruritus occurred simultaneously in two of six individuals in the anifrolumab $600-\mathrm{mg}$ group, but not those in the PBO group. Adverse events were reported by $50 \%(n=9)$ of anifrolumab-treated and $33 \%(n=4)$ of PBO-treated individuals. No serious adverse events were observed. ADAs were detected in only one individual in the anifrolumab 300-mg IV group at the Day-85 assessment.

Serum Concentration-Time Profiles

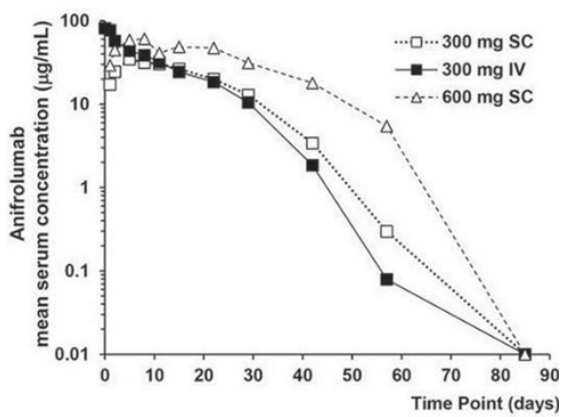

Mean data below limits of detection are plotted as $1 / 2$ of the lower limits of quantification $(0.02 \mu \mathrm{g} / \mathrm{mL})$.

Conclusions: Exposure of anifrolumab $300 \mathrm{mg} \mathrm{SC}$ was approximately $86 \%$ of IV administration, with single SC administrations of anifrolumab being generally well-tolerated in healthy volunteers.

References:

[1] Furie R, et al. Arthritis Rheumatol. 2017;69:376-86.

Disclosure of Interest: R. Tummala Employee of: AstraZeneca, T. Rouse Employee of: AstraZeneca, A. Berglind Shareholder of: AstraZeneca, BMS, Employee of: AstraZeneca, L. Santiago Shareholder of: AstraZeneca, Employee of: Medlmmune

DOI: 10.1136/annrheumdis-2017-eular.2149

\section{SAT0255 A RANDOMIZED, PLACEBO-CONTROLLED, DOUBLE-BLIND, ASCENDING-DOSE, SAFETY STUDY OF CC-220 IN SUBJECTS WITH SYSTEMIC LUPUS ERYTHEMATOSUS}

V.P. Werth ${ }^{1}$, R. Furie ${ }^{2}$, S. Korish ${ }^{3}$, M. Weiswasser ${ }^{3}$, A. Azaryan ${ }^{3}$,

P.H. Schafer ${ }^{3}$, N. Delev ${ }^{3}$, S. Choi ${ }^{3}$, D. Hough ${ }^{3}$. ${ }^{1}$ University of Pennsylvania School of Medicine, Philadelphia; ${ }^{2}$ Northwell Health, Great Neck; ${ }^{3}$ Celgene Corporation, Summit, United States

Background: CC-220 is a CUL4CRBN E3 ubiquitin ligase modulator that binds to cereblon and leads to potent and deep reduction of the transcription factors Ikaros (IKZF1) and Aiolos (IKZF3), which are overexpressed in the peripheral blood of Systemic Lupus Erythematosus (SLE) subjects. This study evaluated the safety, and tolerability of CC-220 in subjects with SLE. Exploratory efficacy assessments were included.

Methods: Subjects with history of SLE $\geq 6$ months and a baseline of hybrid SELENA-SLEDAI (hSS) score $\geq 4$ were randomized to 1 of 4 escalating doses of CC-220 or matching placebo (PBO). The 4 active treatments were CC-220 $0.3 \mathrm{mg}$ QOD, $0.3 \mathrm{mg} \mathrm{QD}, 0.3 \mathrm{mg}$ alternating with $0.6 \mathrm{mg} \mathrm{QD}$, and $0.6 \mathrm{mg} \mathrm{QD}$; subjects were randomized $4: 1$ active to $\mathrm{PBO}$ in each group for 12 weeks of treatment, followed by 12 weeks of observational follow-up and/or long-term extension. Stable doses of corticosteroids ( $\leq 10 \mathrm{mg}$ prednisone or equivalent daily), non-steroidal anti-inflammatory drugs, and antimalarials were permitted. Safety assessments included clinical evaluation of adverse events (AEs), laboratory parameters, electrocardiograms, physical examinations, and overall tolerability. Exploratory efficacy assessments included hSS, Cutaneous Lupus Area and Severity Index (CLASI) skin scores, Physician Global Assessment (PGA), swollen joint counts (SJC), and tender joint counts (TJC).

Results: A total of 42 adult subjects were randomized; 39 subjects were female (93\%); Mean age was 47.2 years; $64 \%$ were White and $31 \%$ were Black or African-American. Mean SLE duration was 9.4 years, with a mean baseline hSS score of 6.6 , CLASI activity score of 9.8 , and PGA score of 1.3. Seventy-nine percent of subjects completed the study; 9 of 42 subjects discontinued, of which 6 subjects discontinued due to an adverse event (AE): 1 in the placebo group and 5 in the 2 highest CC-220 groups combined. No discontinuations were due to lack of efficacy. Four subjects had serious AEs (highest CC-220 doses: $n=2$ [pneumonia]; PBO: $n=2$ ). Three subjects had neutropenia (grade $3: n=2$; grade 1 : $\mathrm{n}=1$ ); 2 subjects in the highest CC-220 dose group had dermatitis, and 1 subject in the $0.3 \mathrm{mg} \mathrm{QD}$ and 1 in the $0.6 \mathrm{mg} \mathrm{QD}$ dose groups had urticaria. Mean reductions in the CLASI activity score at day 85 ranged from 4.3 to 7.8 in the CC-220 treatment groups compared to an increase of 0.4 in the placebo group. More subjects receiving CC-220 had a $\geq 4$-point reduction in hSS score vs PBO by Day $85(22.2 \%>50.0 \%$ vs $12.5 \%)$.

Conclusions: CC-220 was generally well tolerated in this SLE population over 12 weeks of treatment, with neutropenia and dermatitis observed at the highest doses studied. Treatment with CC-220 resulted in a trend toward greater improvement 
in multiple measures of SLE disease activity compared with PBO. These results support further development of CC-220 in SLE patient population.

Disclosure of Interest: V. Werth Grant/research support from: Celgene Corporation, Consultant for: Celgene Corporation, R. Furie Consultant for: Celgene Corporation, S. Korish Shareholder of: Celgene Corporation, Employee of: Celgene Corporation, M. Weiswasser Shareholder of: Celgene Corporation, Employee of: Celgene Corporation, A. Azaryan Shareholder of: Celgene Corporation, Employee of: Celgene Corporation, P. Schafer Shareholder of: Celgene Corporation, Employee of: Celgene Corporation, N. Delev Shareholder of: Celgene Corporation, Employee of: Celgene Corporation, S. Choi Shareholder of: Celgene Corporation, Employee of: Celgene Corporation, D. Hough Shareholder of: Celgene Corporation, Employee of: Celgene Corporation

DOI: 10.1136/annrheumdis-2017-eular.3546

\section{SAT0256 MULTI-TARGET THERAPY WITH MIZORIBINE, TACROLIMUS, AND PREDNISOLONE IN LUPUS NEPHRITIS: ANALYSIS OF 47 CASES}

R. Kawato, R. Rokutanda, M. Kishimoto, M. Okada. Immune-rheumatology center, St.luke hospital, Tokyo, Japan

Background: Mizoribine (MZB), an inosine monophosphate dehydrogenase inhibitor, is an effective glucocorticoid-sparing agent for gromerulonephritis and variety of rheumatic diseases. However, its clinical usefulness as multi-target therapy in patients of lupus nephritis has been unclear.

Objectives: To evaluate efficacy of multi-target therapy with MZB, tacrolimus (TAC) and prednisolone (PSL) for lupus nephritis.

Methods: We extracted all the cases with lupus nephritis treated with PSL, MZB, and TAC during period from 2008/1 to 2016/10. Retrospective medical chart review was performed to collect following data; baseline patient characteristics, dose of PSL, serum creatinine, urine protein, compliment, anti-DNA ab, remission rates, and safety profiles. We define complete remission as urine protein $<0.5$ $\mathrm{g} / \mathrm{gCre}$ and normal serum creatinine, partial remission as urine protein decreasing more than $50 \%$ at baseline, and $<3 \mathrm{~g} / \mathrm{day}$, and normal serum creatinine or increasing within $15 \%$. Patents' records were followed from the beginning of multi-target treatment to 12 months after.

Results: 43 cases (female; $n=37$, male; $n=6$, mean age; 37.4 years old) were included for analysis. Of 30 cases who underwent renal biopsy, 10 cases were classified as class IV nephritis, and 7 cases were classified as class III nephritis. Mean urine protein $(\mathrm{g} / \mathrm{gCr})$ at baseline, at 3 months, and at 6 months are 1.9 , 0.4 and 0.4 , respectively. Mean dose of steroid was $32.8 \mathrm{mg} /$ day at baseline, 9.8 $\mathrm{mg} /$ day at 3 months, and $8.7 \mathrm{mg} /$ day at 6 month. $79.1 \%$ of the patients achieved complete remission at 3 months, and the remission rate was more than $80 \%$ at 6 months and later. As for adverse events, 14 cases had some Infection, 3 out of 14 needed antibiotics treatment. Four cases had renal impairment after starting TAC, all of them recovered after stopping or decreasing dose of TAC.

Conclusions: Multi-target therapy with MZB, TAC and PSL showed good remission rate, and few severe adverse events. We need the further evaluation of long-term efficacy of the therapy.

Disclosure of Interest: None declared

DOI: 10.1136/annrheumdis-2017-eular.3374

\section{SAT0257 "AIMING FOR THE BULLSEYE": RETINAL TOXICITY SCREENING FOR HYDROXYCHLOROQUINE THERAPY - ARE WE HITTING THE TARGET?}

S. Gubbins, J. Steen, A. Gorman, S. Khan, A. Mohammad, K. O'Rourke. Rheumatology, Midlands Regional Hospital Tullamore, Tullamore, Ireland

Background: Hydroxychloroquine ( $\mathrm{HCQ}$ ) is commonly used to treat a variety of rheumatological and inflammatory conditions such as RA, SLE and Sjogrens. Retinal toxicity is a well known complication of HCQ therapy. Initially, the incidence of retinal toxicity was believed to be quite minimal1. However, subsequent large epidemiological studies have highlighted its much greater prevalence-up to $7.5 \%$ in certain populations 2,3 . Furthermore, the visual toxicity is typically permanent with possible progression up to years after drug cessation and with no current treatment4. Detection at an early stage has been shown to be crucial in preserving central vision and foveal architecture5. As a result ophthalmological screening is an essential consideration for any patient on HCQ therapy.

In view of the updated scientific data available on toxicity prevalence and the benefits of screening, the American Academy of Ophthalmology (AAO) have updated their screening recommendations for 20166. This includes baseline fundus examination to rule out pre-existing maculopathy and annual screening after 5 years of treatment.

Objectives: To investigate compliance with the AAO Recommendation on Screening for Chloroquine and Hydroxychloroquine Retinopathy (2016)6 in a regional rheumatology centre.

Methods: A retrospective analysis was undertaken of the electronic patient records of 161 patients with a diagnosis of connective tissue disease at a single regional centre assessing level of antimalarial usage, adherence to screening guidelines and level of documented toxicity.

Results: Of our cohort, 91 (56\%) were on regular HCQ. Of these, 12 had received routine ophthalmology screening and 4 developed documented ocular toxicity.
15 suffered non-ophthalmic toxicity requiring therapy cessation. 46 had been on therapy greater than 5 years and of these $9(21.5 \%)$ had been screened.

Conclusions: Our analysis revealed significant deficiencies in routine screening and demonstrated a high level of toxicity in patients who were routinely screened. This indicates that there may be a significant number of patients with toxicity that is not being picked up and reinforces the importance of screening. We would recommend a more streamlined referral pathway for rheumatology patients on $\mathrm{HCQ}$ and hypothesise that this would lead to greater and earlier pick-up of ocular toxicity in this population, ultimately reducing morbidity in this population.

References:

[1] Elman A, Gullberg R Nilsson E, et al. Chloroquine retinopathy in patients with rheumatoid arthritis. Scand J Rheumatol. 1976;5(3):161-66.

[2] Melles RB, Marmor MF. The risk of toxic retinopathy in patients on long-term hydroxychloroquine therapy. JAMA Ophthalmol 2014;132:1453-60.

[3] Levy GD, Munz SJ, Paschal J, et al. Incidence of hydroxychloroquine retinopathy in a large multicentered outpatient practice. Arthritis Rheum. 1997 Aug;40(8):1482-6.

[4] Marmor MF, Hu J. Effect of disease stage on progression of hydroxychloroquine retinopathy. JAMA Ophthalmol 2014;132:1105-12.

[5] Marmor MF, Hu J. Effect of disease stage on progression of hydroxychloroquine retinopathy. JAMA Ophthalmic 2014;132:1105-12.

[6] Michael F, Marmor MD et al, Recommendations on Screening for Chloroquine and Hydroxychloroquine Retinopathy (2016 Revision) Ophthalmology Volume 123, Number 6, June 2016.

Disclosure of Interest: None declared

DOI: 10.1136/annrheumdis-2017-eular.6547

\section{SAT0258 SYNERGETIC B-CELL IMMUNOMODULATION WITH RITUXIMAB AND BELIMUMAB IS CLINICALLY EFFECTIVE IN SEVERE AND REFRACTORY SYSTEMIC LUPUS ERYTHEMATOSUS - THE SYNBIOSE PROOF-OF-CONCEPT STUDY}

T. Kraaii ${ }^{1}$, S.W. Kamerling ${ }^{1}$, E. de Rooij ${ }^{1}$, P.L. van Daele ${ }^{2}$, I. Bajema ${ }^{3}$, O.W. Bredewold ${ }^{1}$, T.W. Huizinga ${ }^{4}$, T.J. Rabelink ${ }^{1}$, C. van Kooten ${ }^{1}$, Y.K. Teng ${ }^{1}$. ${ }^{1}$ Nephrology, LUMC, Leiden; ${ }^{2}$ Immunology, Erasmus MC, Rotterdam; ${ }^{3}$ Pathology; ${ }^{4}$ Rheumatology, LUMC, Leiden, Netherlands

Background: The pathogenesis of systemic lupus erythematosus (SLE) is characterized by B-cell hyperactivity leading to autoreactive plasma cells (PCs) that produce pathogenic autoantibodies and contribute to onset and maintenance of SLE. Therefore, novel treatment strategies in SLE are aimed at targeting autoreactive PCs. Rituximab (RTX) is used as off-label treatment in SLE and depletes CD20+ B-cells effectively, but is unable to deplete CD20- PCs. Belimumab (BLM), an anti-BAFF (B-cell activating factor) antibody, demonstrated efficacy in SLE as add-on therapy and resulted in reduction of B-cells and PCs. Objectives: Because RTX+BLM has potential to target autoreactive PCs, we designed an open-label, proof-of-concept study to assess the clinical efficacy and safety of RTX+BLM in severe, refractory SLE patients.

Methods: A phase 2 proof-of-concept study, the SynBioSe study, was set up to treat severe, serologically active and refractory SLE patients with RTX following BLM. The following endpoints were assessed at 24 weeks: a) clinical efficacy by SLEDAl; b) immunological effects on B-cells and PCs by high sensitivity flow cytometry, immunoglobulins and autoantibody levels, and c) safety parameters. Results: The study included 11 severe, refractory SLE patients of which 10 had lupus nephritis and 1 patient had neuropsychiatric lupus with a median SLEDAI of 18 [range 8-29]. Treatment with RTX+BLM led to clinically significant improvement to a median SLEDAI score of $2[0-13](p=0.002)$. Low disease activity (SLEDAI <4) was achieved in $80 \%$. Clinical response was achieved while tapering steroids from a median dose of $60[0-60]$ to $7.5 \mathrm{mg}[0-12.5]$ and concomitant mycophenolate (MMF) was tapered to zero in all patients. With respect to safety, 23 adverse events (AEs) were reported of which none were serious AEs, 8 mild infections and 2 hypogammaglobulinemia. Complement usage was restored in all patients with a median increase in C3 of 33\% [range 8-133] and in C4 levels of 39\% [9-266]. RTX+BLM led to reduction of anti-dsDNA antibodies, from median $120 \mathrm{IU} / \mathrm{ml}[18-505]$ to $35 \mathrm{IU} / \mathrm{ml}$ [10-374] $(\mathrm{p}=0.012)$. CD19+ B-cells were depleted throughout the complete follow up (from median $206^{*} 10^{6}$ cells $/ L$ [range $\left.21^{*} 10^{6}-511 \times 10^{6}\right]$ at screening to $5 \times 10^{6}\left[0-24 \times 10^{6}\right]$ at 24 weeks, $p=0.004$ ), including transitional B-cells (from median $1.3 \times 10^{3}$ cells/L $\left[1-1.3 \times 10^{4}\right]$ at screening to $98.5\left[0-3.4 \times 10^{3}\right]$ at 24 weeks, $\left.p=N S\right)$. Importantly, total immunoglobulin levels only declined temporarily. The latter was further corroborated by transient reductions in CD3-CD38brCD27brCD20- PCs from median $1.6 \times 10^{6}$ cells $/ \mathrm{L}\left[5.6 \times 10^{3}-1.1 \times 10^{7}\right]$ to $1.5 \times 10^{5}$ cells $/ L$ [5.3 $\left.\times 10^{4}-1.3 \times 10^{7}\right]$ at 12 weeks $(p=N S)$. At 24 weeks, these PCs were restored to baseline levels $\left(1.4 \times 10^{6}\right.$ cells $\left./ \mathrm{L}\left[9.2 \times 10^{4}-3 \times 10^{7}\right]\right)$.

Conclusions: This is the first study to demonstrate that RTX+BLM effectively reduced disease activity in severe, refractory SLE without raising major safety issues, while reducing other immunosuppressant drugs. Autoreactive PCs were specifically targeted by RTX+BLM. Therefore, combination therapy of RTX+BLM is a promising strategy in severe SLE.

Trial registration:ClinicalTrials.gov NCT02284984

Disclosure of Interest: None declared

DOI: 10.1136/annrheumdis-2017-eular.2364 